\title{
Tradução e adaptação transcultural da escala de atividade física para pessoas com deficiência física (PASIPD BR)
}

\author{
Translation and cross-cultural adaptation of physical activity scale for \\ individuals with physical disabilities (PASIPD BR)
}

Traducción y adaptación transcultural de la escala de actividad física para personas con discapacidad física (PASIPD BR)

\section{Douglas Felipe Veroni Rodrigues}

Especialista pela Universidade Estadual Paulista Júlio Mesquita Filho, Marília, São Paulo, Brasil. fisioveroni@hotmail.com

ORCID - https://orcid.org/0000-0003-2989-0556

Fernanda Carolina Toledo da Silva

Doutora pela Universidade Estadual Paulista Júlio Mesquita Filho, Marília, São Paulo, Brasil.

nanda tol@hotmail.com

ORCID - https://orcid.org/0000-0001-7959-5593

Richard Avery Washburn

Cientista sênior na Universidade do Kansas, Kansas City, Kansas, Estados Unidos da América. rwashburn@ku.edu

ORCID - https://orcid.org/0000-0002-8484-0669

Lígia Maria Presumido Braccialli

Professora doutora na Universidade Estadual Paulista Júlio Mesquita Filho, Marília, São Paulo, Brasil. ligiapresumido@hotmail.com

ORCID - https://orcid.org/0000-0002-2540-3725

Recebido em 19 novembro 2019

Aprovado em 21 de setembro de 2020

Publicado em 26 de outubro de 2020

\section{RESUMO}

A prática de atividade física regular é importante para proporcionar à pessoa com deficiência física bem-estar e melhor qualidade de vida. Observa-se uma carência de instrumentos padronizados e específicos na língua portuguesa para avaliação do nível de atividade física direcionados a essa população. O estudo teve como objetivo traduzir e adequar culturalmente o instrumento Physical Activity Scale for Individuals with Physical Disabilities - PASIPD para o português do Brasil. Para o processo de tradução e adaptação transcultural da escala, foram realizadas cinco etapas: 1) tradução inicial; 2) síntese da tradução; 3) retrotradução; 4) equivalência semântica e operacional; e, 5) Préteste. Na equivalência semântica, 7 itens obtiveram índice de concordância abaixo de $70 \%$. No pré-teste o instrumento foi testado com adultos com deficiência física, por seis profissionais especialistas na área de Reabilitação Física. Nessa etapa apenas um item teve índice de concordância abaixo de $70 \%$. Após a adequação dos itens que apresentaram dificuldade a escala foi considerada adequada culturalmente para verificar o nível de atividade física em indivíduos com deficiência física no Brasil.

Palavras-chave: Atividade física; Deficiência Física; instrumento de avaliação. 
http://dx.doi.org/10.5902/1984686X41214

\section{ABSTRACT}

The practice of regular physical activity is important to provide well-being and better quality of life to person with physical disability. There is a lack of standardized and specific instruments in Portuguese to assess the level of physical activity directed at this population. The purpose of this study was to translate and culturally adapt the Physical Activity Scale for Individuals with Physical Disabilities (PASIPD) to Brazilian Portuguese. For the process of transcultural translation and adaptation of the scale, five steps were performed: 1) initial translation; 2) translation synthesis; 3) back-translation; 4) semantic and operational equivalence; 5) Pre-test. In the semantic equivalence, 7 items obtained a concordance index below $70 \%$. In the pre-test the instrument was tested with adults with physical disabilities by six professionals specialized in Physical Rehabilitation. In this stage, only one item had a concordance index below $70 \%$. After adjusting the items that presented difficulty, the scale was considered culturally adequate to verify the level of physical activity in individuals with physical disabilities in Brazil.

Keywords: Physical activity; Physical Disability; evaluation instrument.

\section{RESUMEN}

La práctica de actividad física regular es importante para proporcionar bienestar y una mejor calidad de vida a las personas con discapacidad física. Hay una falta de instrumentos estandarizados y específicos en portugués para evaluar el nivel de actividad física dirigido a esta población. El propósito de este estúdio fue traducir y adaptar culturalmente la Escala de Activitad Física para Personas com Discapacidades Físicas (PASIPD) al portugués brasileño. Para el proceso de traducción y adaptación transcultural de la escala, se realizaron cinco pasos: 1) traducción incial; 2) síntesis de la traducción; 3) traducción inversa; 4) equivalencia semántica y operativa; 5) prueba previa. En la equivalencia semántica, 7 ítems obtuvieron un índice de concordancia inferior al 70\%. Em la prueba previa, el instrumento fue probado com adultos con discapacidades físicas por seis profesionales especializados en el campo de la rehabilitación física. En esta etapa, solo un ítem tenía un índice de concordancia inferior al 70\%. Después de ajustar los ítems que presentaban dificultad, la escala se consideró culturalmente adecuada para verificar el nivel de actividad física en personas con discapacidades físicas en Brasil.

Palabras clave: Actividad física; Discapacidad Física; instrumento de evaluación.

\section{Introdução}

Os benefícios físicos, psicológicos e sociais da prática de atividade física para pessoas com deficiência física têm sido amplamente documentados. Os benefícios que a referida atividade proporciona são facilmente percebidos, como a prevenção de doenças associadas ao sedentarismo, melhorias no aspecto físico-motor como aumento na composição corporal, melhora na habilidade motora grossa, equilíbrio, coordenação e aptidão cardiovascular, melhora na saúde psicológica e social (SHIELDS; SYNNOT, 2016). 
http://dx.doi.org/10.5902/1984686X41214

Apesar dos benefícios da prática de atividade física sistemática, as pessoas com deficiência física são menos ativas em comparação com a população em geral (CARROLL, et al., 2014; EISENBERG; VANDERBOM; VASUDEVAN, 2017).

Promover um estilo de vida ativo é de grande importância para pessoas com deficiência física já que elas possuem maior risco de desenvolver condições de saúde secundárias por conta de sua deficiência (CARROLL, et al., 2014; SHAW et al., 2017).

Autores têm relatado que programas de intervenção para a prática de atividade física promovem modificações comportamentais positivas em pessoas com deficiência. No entanto, ocorre o risco de viés durante a análise da eficácia desses programas, decorrente da falta de instrumento padronizado para avaliação (CASTRO et al., 2017).

Os efeitos de programas de atividade física para pessoas com deficiência devem ser verificados por meio de métodos e instrumentos validados e confiáveis de avaliação direta e indireta associados. As ferramentas indiretas referem-se aos instrumentos, como questionários, escalas, diários de atividades, cujas informações são obtidas por meio de autorrelato, que, geralmente, exigem tamanho de amostra maior ao avaliar mudanças ao longo do tempo ou diferenças entre os grupos. Os métodos diretos, por outro lado, avaliam as mudanças fisiológicas reais que se correlacionam com a atividade física. Ferramentas diretas são consideradas mais objetivas e precisas (JIMENEZ-MORENO, et al., 2017).

Dentre as ferramentas de medidas indiretas específicas para avaliar a prática de atividade física em indivíduos com deficiência física destaca-se a Physical Activity Scale for Individuals with Physical Disabilities - PASIPD, instrumento originalmente desenvolvido e validado para a língua inglesa (WASHBURN et al., 2002). Posteriormente, o instrumento foi traduzido, adaptado e validado para o holandês (VAN DER PLOEG et al., 2007), para a língua malaia (MAT ROSLY et al., 2019), para o francês (DRIGNY, 2019), para o turco (ULAŞ; TOPUZ; DINÇ HORASAN, 2019), para o Norueguês (SKATTEBOE, et al., 2016).

Avaliações da validade dessa escala para a população com deficiência física concluíram que: apresenta uma boa validade de construto na língua inglesa (WASHBURN et al., 2002), a validade de seu critério é semelhante aos questionários de atividade física bem estabelecidos da atividade da população em geral (VAN DER PLOEG et al., 2007), tem correlação moderada entre o escore PASIPD com uma a lista de atividades previamente determinadas (DE GROOT et al., 2010), apresenta baixa correlação com ferramentas de medidas diretas, porém instrumentos utilizados com a população sem deficiência também apresenta (VAN DER BERG-EMONS et al., 2011). 
http://dx.doi.org/10.5902/1984686X41214

A escala PASIPD é composta por 13 questões que registram a quantidade de dias por semana e horas por dia que o respondente com deficiência física participou em atividades físicas de lazer, domésticas e ocupacionais nos últimos 7 dias. Cada uma dessas questões é dividida em duas partes. Primeiro, o respondente preenche a frequência com que a atividade foi realizada semanalmente, na segunda parte é preenchido o número de horas por dia despendidas em cada atividade desenvolvida. Para o cálculo do escore, a questão 1 não é pontuada, e, para as demais, há itens multiplicadores e, posteriormente, uma tabela para a conversão dos valores que serão pontuados conforme a frequência de dias da semana e do número de horas que a atividade física foi realizada. Em seguida, é realizada a somatória a fim de se obter o escore total em Equivalente Metabólico - MET horas/dia, que equivale ao gasto energético diário em atividades físicas. O instrumento tem como valor máximo 199,5 MET hr/d. Quanto maior a pontuação obtida no escore total, maior o nível de atividade física do respondente (SILVA, 2019).

Uma vez que o desenvolvimento de um instrumento inclui diversos custos, a adaptação cultural é uma maneira confiável de usar traduções de instrumentos de autorrelato estrangeiros. Dentre as facilidades, pode-se destacar o tempo gasto e esforços poupados pelos pesquisadores e menores custos. A adaptação transcultural também promove a possibilidade de comparar os resultados entre diferentes amostras, bem como a realização de estudos transculturais utilizando o mesmo instrumento (SARDINHA et al., 2010).

O estudo teve como objetivo realizar a tradução e adaptação transcultural da escala Physical Activity Scale for Individuals with Physical Disabilities (PASIPD) para o português do Brasil.

\section{Métodos}

Foi realizado um estudo do tipo metodológico, que se caracteriza pelo desenvolvimento, validação e avaliação de instrumentos e métodos de pesquisa. Esse estudo integra uma pesquisa maior e foi aprovado pelo Comitê de Ética em Pesquisa da Faculdade de Filosofia e Ciências de Marilia - UNESP, com parecer número 1.503.491. Para a realização da tradução e adaptação transcultural para o português do Brasil do instrumento PASIPD foi obtida autorização formal do autor da escala. Todos os participantes de todas as etapas do estudo assinaram o Termo de Consentimento Esclarecido. 
http://dx.doi.org/10.5902/1984686X41214

\section{Participantes}

Os participantes de cada etapa do estudo foram apresentados na Figura 1. Na Etapa 01-Tradução Inicial, um dos participantes tinha como língua nativa o português e era tradutor juramentado para a língua inglesa, o outro tradutor tinha formação em Educação Física com ampla experiência na área de atividade física adaptada e fluência na língua inglesa. Os participantes da Etapa 02 - Síntese da tradução, foram três pesquisadores especialistas em Atividade Física Adaptada e com experiência na língua inglesa, sendo dois com formação em Fisioterapia e um em Educação Física, com uma média de 15 anos de experiência na área. Na Etapa 03 - Retrotradução, foi participante um tradutor nativo da língua inglesa e tradutor juramentado para língua portuguesa do Brasil. A Etapa 04 - Análise semântica e operacional, foi composta por cinco juízes especialistas na área de Atividade Física Adaptada e Educação Especial, com Doutorado em Educação Especial ou Educação Física. Já na Etapa 05 - Pré-teste, participaram seis profissionais especialistas na área de Reabilitação Física, com experiência de, pelo menos, um ano, sendo cinco fisioterapeutas com especialização em Reabilitação e Tecnologia e uma professora de Educação Física, mestre em Educação e seis indivíduos adultos com deficiência física. Os indivíduos com deficiência física tinham diagnóstico de: 1 Lesão Medular e Amputação de membro inferior, 1 Tumor Intramedular, 2 Esclerose Múltipla, 1 Miopatia Congênita Desproporção Fibras e 1 Amputação, com idade entre 18 e 40 anos.

Figura 1 - Participantes de cada etapa do estudo

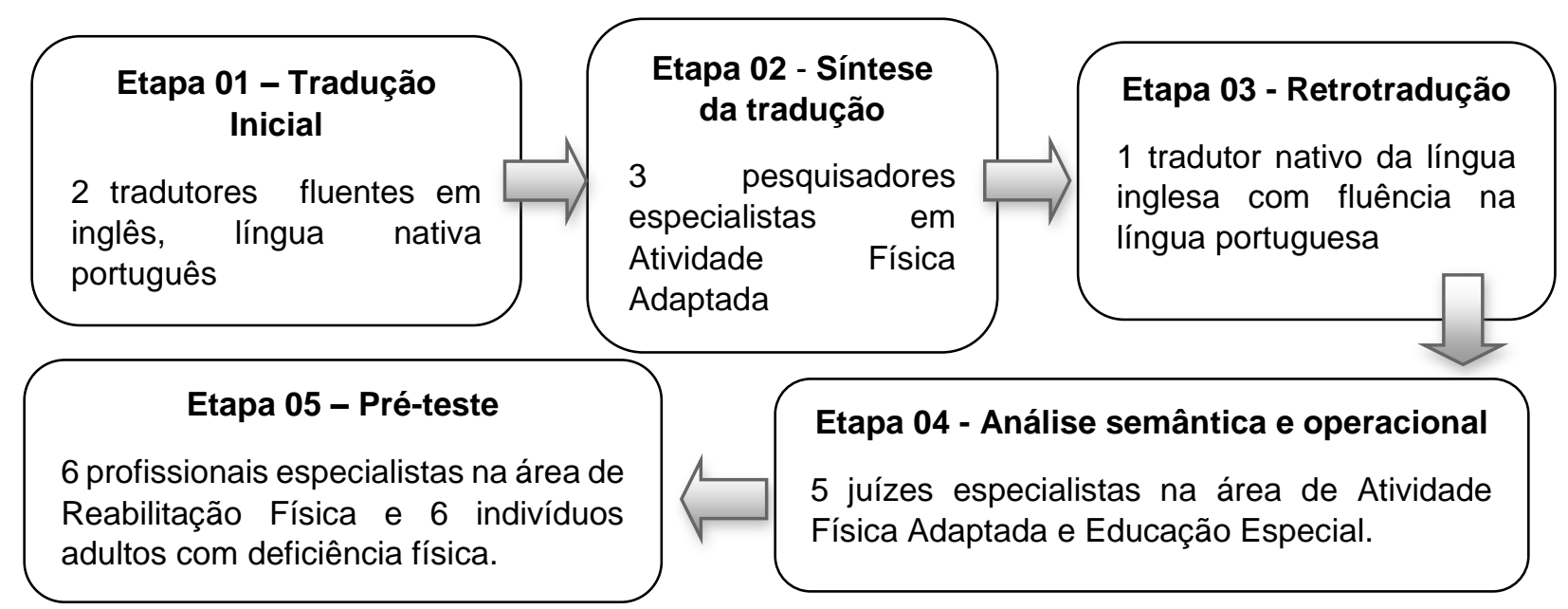

Fonte: Elaborado pelos autores (2019). 
http://dx.doi.org/10.5902/1984686X41214

\section{Procedimentos}

Para o processo de tradução e adaptação transcultural da escala PASIPD para o português, foram realizadas as seguintes etapas: 1) Tradução Inicial; 2) Síntese da Tradução; 3) Retrotradução; 4) Avaliação semântica e Operacional; 5) Pré-teste. Essas etapas são recomendadas por diversos autores (GUILLEMIN; BOMBARDIER; BEATON, 1993; BEATON et al., 2000; MELCHIORS; CORRER; FERNÁNDEZ-LLIMOS, 2007; COSTER; MANCINI, 2015).

Na Etapa 1 - Tradução Inicial, dois tradutores fluentes na língua inglesa, e que tinham o português como língua nativa, realizaram a tradução da versão original do inglês para o português do Brasil. O tradutor 1 (T1) era tradutor juramentado para a língua inglesa com experiência na tradução de instrumentos e o tradutor 2 (T2) era um profissional formado em Educação Física, fluente na língua inglesa e na área da Atividade Física Adaptada. O cuidado na escolha dos tradutores foi importante, uma vez que não se deseja a tradução literal do instrumento, mas, uma versão compreensível na nova língua. Autores tem recomendado que o tradutor tenha como idioma nativo aquele para qual o instrumento será traduzido, e que tenha alguma familiaridade com a área e com o instrumento (COSTER; MANCINI, 2015).

Os tradutores trabalharam de forma independente, não se conheciam e não tiveram contato durante todo o processo. Nesta etapa, tivemos como resultado a elaboração de duas versões independentes, T1 e T2, do instrumento para a língua portuguesa do Brasil.

$\mathrm{Na}$ etapa seguinte foi realizada a síntese da tradução por uma equipe formada por três pesquisadores com experiência na área de Atividade Física Adaptada, com domínio da língua inglesa e experientes na tradução e adaptação de instrumentos, e que tinham o português do Brasil como língua nativa. Os pesquisadores discutiram cada item da escala, analisaram qual a melhor tradução, verificaram se deveria haver adequações dos termos e exemplos para a nova cultura. Nessa etapa, os pesquisadores, também, realizaram a equivalência conceitual e de itens. Os autores Reichenheim e Moraes (2007) orientam que, para obter essas equivalências, os especialistas devem verificar se os diferentes domínios existentes no instrumento original são relevantes e pertinentes ao novo contexto ao qual foi adaptado. Ao final dessa etapa obteve-se a versão 1 do instrumento na língua portuguesa do Brasil.

Ao término da primeira versão da tradução do instrumento, o texto foi enviado a um profissional nativo da língua inglesa e fluente em português para realizar a retrotradução 
para o idioma de origem. A retrotradução é fundamental para garantir que todo o conteúdo do instrumento, de um ponto de vista conceitual, não tenha se modificado na fase de tradução e, portanto, é a chave para o estabelecimento da equivalência semântica do instrumento (PICON et al., 2005).

A retrotradução da versão 1 foi enviada ao autor do instrumento para verificação da adequação, identificação e correção de possíveis discrepâncias existentes com a versão original. Após as considerações do autor, o instrumento retornou à equipe de pesquisadores, que tinha feito a síntese da tradução, que realizou as modificações necessárias que deu origem a versão 2 do instrumento em língua portuguesa do Brasil.

Para a verificação da equivalência semântica e operacional foi elaborado e encaminhado para cinco juízes especialistas em Atividade Física Adaptada um Protocolo de Análise de Equivalência Semântica e Operacional.

$\mathrm{Na}$ última etapa foi realizado o pré-teste do instrumento por um grupo composto por cinco fisioterapeutas e um professor de educação física. Essa etapa teve como objetivo identificar o nível de compreensão, entendimento, erros, desvios de tradução e possíveis sugestões a todos os itens do instrumento.

O pré-teste consistiu na aplicação do instrumento, por profissionais com experiência na área, em pessoas adultas com deficiência física. Cada profissional realizou a aplicação da escala em formato de entrevista com uma pessoa com deficiência física. O profissional realizava a leitura de cada item do instrumento para a pessoa com deficiência física e ela respondia à questão formulada no instrumento, o avaliador preenchia o resultado no formulário e fazia anotações se houve alguma dificuldade de compreensão e qual a dificuldade. $O$ avaliador podia também realizar sugestões sobre modificações que considerava importante.

\section{Procedimentos de análise dos dados}

As respostas dos protocolos foram organizadas e, em seguida, realizado o índice de concordância entre os juízes. Para a análise do índice de concordância foi utilizada a fórmula preconizada por Fagundes (1999). O índice de concordância foi considerado aceitável quando foi igual ou superior a $70 \%$. Os itens que não atingiram esse índice foram reformulados. 
http://dx.doi.org/10.5902/1984686X41214

\section{Resultados}

Os resultados foram apresentados em quatro categorias equivalente às etapas do processo de tradução: a) síntese da tradução; b) equivalência semântica; c) equivalência operacional; e, d) pré-teste. Procuramos descrever cada categoria melhor nos parágrafos seguintes.

\section{A - Síntese da tradução}

Na etapa Síntese da tradução os pesquisadores, ao realizarem a análise, sugeriram modificar ou excluir alguns exemplos de atividade física que não ocorriam rotineiramente no contexto brasileiro, como na questão 10, o exemplo "remover neve" foi excluído.

\section{B - Equivalência semântica}

Em relação a equivalência semântica, seis itens do instrumento tiveram $80 \%$ de concordância, seis itens tiveram índice de Concordância em 60\% e um item teve 40\% de Concordância (Tabela 1). Os itens que tiveram baixa concordância foram revistos pela equipe de especialistas que realizaram a síntese da tradução e modificaram conforme as sugestões dos juízes, uma vez que as sugestões estavam relacionadas à ortografia da língua portuguesa.

Tabela 1 - Índice de concordância da Equivalência semântica da versão original com a versão em port. do Brasil

Versão português do Brasil

1. Durante os últimos 7 dias, com que frequência você realizou atividades sentado (a) ou deitado (a), tais como ler, assistir TV, jogar no computador/ celular/ tablet ou fazer trabalhos manuais?
IC Modificação após sugestões dos juízes

(continua)
2. Durante os últimos 7 dias, com que frequência você andou ou impulsionou a cadeira de rodas fora de sua casa que não para se exercitar. Por exemplo, ir para o trabalho ou para aula, sair com o cachorro para fazer compras, ou outros serviços?

3. Durante os últimos 7 dias, com que frequência você participou de esportes ou atividades recreativas leves, tais como boliche, golfe com carrinho, pesca, jogo de dardo, bilhar ou sinuca, exercícios terapêuticos (fisioterapia ou terapia ocupacional, alongamento, uso de aparelho para ficar em pé) ou outras atividades similares?
60 1. Nos últimos 7 dias, com que frequência você realizou atividades sem esforço físico, tais como ler, assistir TV, jogar no computador/ celular/ tablet ou fazer trabalhos manuais?

60 2. Nos últimos 7 dias, com que frequência você caminhou ou impulsionou a cadeira de rodas fora de sua casa sem a finalidade de realizar exercícios. Por exemplo, ir para o trabalho ou para aula, sair com o cachorro para fazer compras, ou outras atividades?

60 3. Nos últimos 7 dias, com que frequência você participou de esportes ou atividades recreativas leves, tais como boliche, golfe com carrinho, pesca, jogo de dardo, bilhar ou sinuca, exercícios terapêuticos (fisioterapia ou terapia ocupacional, alongamento, uso de aparelho para ficar em pé) ou outras atividades similares? 
http://dx.doi.org/10.5902/1984686X41214

Tabela 1 - Índice de concordância da Equivalência semântica da versão original com a versão em port. do Brasil

(conclusão)

Versão português do Brasil

4. Durante os últimos 7 dias, com que frequência você participou de esportes ou atividades recreativas moderadas, tais como partidas de tênis em dupla, softbol, golfe sem carrinho, dança de salão, andando ou impulsionando cadeira de rodas por lazer ou outras atividades similares?

5. Durante os últimos 7 dias, com que frequência você participou de esportes ou atividades recreativas pesadas tais como corrida, corrida de cadeira de rodas (treinamento), trilhas impulsionando a cadeira de rodas, natação, dança aeróbica, cicloergômetro, ciclismo (pernas ou braços), partida de tênis individual, rugby, basquete, andar com muletas ou andadores, ou outras atividades similares?
IC Modificação após sugestões dos juízes

60 4. Nos últimos 7 dias, com que frequência você participou de esportes ou atividades recreativas moderadas, tais como partidas de tênis em dupla, softbol/beisebol, golfe sem carrinho, dança de salão, impulsionando cadeira de rodas por lazer ou outras atividades similares?

60 5. Nos últimos 7 dias, com que frequência você participou de esportes ou atividades recreativas intensas tais como corrida, corrida de cadeira de rodas (treinamento), trilhas impulsionando a cadeira de rodas, natação, dança aeróbica, cicloergômetro, ciclismo (pernas ou braços), partida de tênis individual, rúgbi, basquete, andar com muletas ou andadores, ou outras atividades similares?

6. Durante os últimos 7 dias, com que frequência você 806 . Nos últimos 7 dias, com que frequência você realizou qualquer exercício especificamente para aumento de força muscular ou resistência tais como levantamento de pesos; flexões; flexões em barra fixa ou em barras paralelas; levantamentos (push-up) na cadeira de rodas, etc.? realizou qualquer exercício especificamente para aumento de força muscular ou resistência tais como levantamento de pesos; flexões; flexões em barra fixa ou em barras paralelas; levantamentos (push-up) na cadeira de rodas etc.?

7. Durante os últimos 7 dias, com que frequência você 80 7. Nos últimos 7 dias, com que frequência você fez fez atividades domésticas leves, tais como tirar o pó, varrer o chão ou lavar louça? atividades domésticas leves, tais como tirar o pó, varrer o chão ou lavar louça?

8. Durante os últimos 7 dias, com que frequência você 80 8. Nos últimos 7 dias, com que frequência você fez fez atividades domésticas pesadas, tais como passar aspirador, esfregar o chão, lavar janelas ou paredes, etc.? atividades domésticas pesadas, tais como passar aspirador, esfregar o chão, lavar janelas ou paredes etc.?

9. Durante os últimos 7 dias, com que frequência você 80 9. Nos últimos 7 dias, com que frequência você fez fez reparos em casa como marcenaria, pintura, reparos em móveis, trabalhos elétricos, etc.? reparos em casa como marcenaria, pintura, reparos em móveis, trabalhos elétricos etc.?

10. Durante os últimos 7 dias, com que frequência você 60 10. Nos últimos 7 dias, com que frequência você cuidou trabalhou no gramado ou cuidou do quintal, tal como cortando grama, rastelando folhas, aparando árvores ou arbustos, ou cortando madeira, etc.? do gramado ou do quintal, como por exemplo cortando grama, rastelando folhas, aparando árvores ou arbustos, ou cortando madeira etc.?

11. Durante os últimos 7 dias, com que frequência você 40 11. Nos últimos 7 dias, com que frequência você passou fora de casa trabalhando no jardim? passou fora de casa cuidando do jardim?

12. Durante os últimos 7 dias, com que frequência você 80 12. Nos últimos 7 dias, com que frequência você cuidou de outra pessoa, tal como crianças, um esposo (a) dependente, ou outro adulto? cuidou de outra pessoa, tal como crianças, um esposo (a) dependente, ou outro adulto?

13. Durante os últimos 7 dias, com que frequência você 80 13. Nos últimos 7 dias, com que frequência você trabalhou com remuneração ou como voluntário? (Exclua trabalho que envolva principalmente ficar sentado com pouco movimento dos braços, tais como trabalho leve de escritório, trabalho no computador, trabalho leve de linha de produção, dirigir um ônibus ou uma van, etc.). trabalhou com remuneração ou como voluntário? (Exclua trabalho que envolva principalmente ficar sentado com pouco movimento dos braços, tais como trabalho leve de escritório, trabalho no computador, trabalho leve de linha de produção, dirigir um ônibus ou uma van etc.).

Fonte: Elaborado pelos autores (2019). 
http://dx.doi.org/10.5902/1984686X41214

\section{C - Equivalência operacional}

Quanto à equivalência operacional todos os itens tiveram Índice de Concordância de 100\%, não sendo necessária nenhuma modificação.

\section{D - Pré-teste}

Os resultados do índice de concordância entre os profissionais que aplicaram o questionário com as pessoas com deficiência física foram apresentados na Tabela 2.

Tabela 2 - Índice de concordância do pré-teste

\begin{tabular}{ll}
\hline Itens & IC \\
\hline 1. Nos últimos 7 dias, com que frequência você realizou atividades sem esforço físico, tais como ler, assistir & 100 \\
TV, jogar no computador/ celular/ tablet ou fazer trabalhos manuais? &
\end{tabular}

2. Nos últimos 7 dias, com que frequência você caminhou ou impulsionou a cadeira de rodas fora de sua 100 casa sem a finalidade de realizar exercícios. Por exemplo, ir para o trabalho ou para aula, sair com o cachorro para fazer compras, ou outras atividades?

3. Nos últimos 7 dias, com que frequência você participou de esportes ou atividades recreativas leves, tais 83 como boliche, golfe com carrinho, pesca, jogo de dardo, bilhar ou sinuca, exercícios terapêuticos (fisioterapia ou terapia ocupacional, alongamento, uso de aparelho para ficar em pé) ou outras atividades similares?

4. Nos últimos 7 dias, com que frequência você participou de esportes ou atividades recreativas moderadas, 83 tais como partidas de tênis em dupla, softbol/beisebol, golfe sem carrinho, dança de salão, impulsionando cadeira de rodas por lazer ou outras atividades similares?

5. Nos últimos 7 dias, com que frequência você participou de esportes ou atividades recreativas intensas tais 83 como corrida, corrida de cadeira de rodas (treinamento), trilhas impulsionando a cadeira de rodas, natação, dança aeróbica, cicloergômetro, ciclismo (pernas ou braços), partida de tênis individual, rúgbi, basquete, andar com muletas ou andadores, ou outras atividades similares?

6. Nos últimos 7 dias, com que frequência você realizou qualquer exercício especificamente para aumento 67 de força muscular ou resistência tais como levantamento de pesos; flexões; flexões em barra fixa ou em barras paralelas; levantamentos (push-up) na cadeira de rodas etc.?

7. Nos últimos 7 dias, com que frequência você fez atividades domésticas leves, tais como tirar o pó, varrer 100 o chão ou lavar louça?

8. Nos últimos 7 dias, com que frequência você fez atividades domésticas pesadas, tais como passar 100 aspirador, esfregar o chão, lavar janelas ou paredes etc.?

9. Nos últimos 7 dias, com que frequência você fez reparos em casa como marcenaria, pintura, reparos em 83 móveis, trabalhos elétricos etc.?

10. Nos últimos 7 dias, com que frequência você cuidou do gramado ou do quintal, como por exemplo 100 cortando grama, rastelando folhas, aparando árvores ou arbustos, ou cortando madeira etc.?
11. Nos últimos 7 dias, com que frequência você passou fora de casa cuidando do jardim?
12. Nos últimos 7 dias, com que frequência você cuidou de outra pessoa, tal como crianças, um esposo (a) 100 dependente, ou outro adulto?

13. Nos últimos 7 dias, com que frequência você trabalhou com remuneração ou como voluntário? (Exclua 100 trabalho que envolva principalmente ficar sentado com pouco movimento dos braços, tais como trabalho leve de escritório, trabalho no computador, trabalho leve de linha de produção, dirigir um ônibus ou uma van etc.).

Fonte: Elaborado pelos autores (2019). 
http://dx.doi.org/10.5902/1984686X41214

Apenas o item 6 do instrumento teve índice de concordância em 67\%, valor abaixo da porcentagem considerada adequada. Este item está relacionado a exercícios realizados, especificamente, para aumento de força muscular ou resistência. Os respondentes tiveram dúvidas neste item, uma vez que este tipo de exercício eles realizavam durante os atendimentos de fisioterapia, respondido no item 3. Foi acrescentado, nesse item, explicação que as atividades poderiam ser consideradas atividades realizadas durante a fisioterapia, desde que os exercícios realizados eram correspondentes ao aumento de força muscular. $\mathrm{O}$ instrumento tem como objetivo mensurar o gasto energético diário em atividades físicas, independentemente do local onde o exercício foi realizado.

As questões Q1, Q3, Q4, Q5, Q9 e Q11 resultaram em um Índice de Concordância em 83\%, não havendo assim a necessidade de reformulação dos mesmos. Os demais itens do instrumento obtiveram um resultado de 100\% de Concordância no Pré-Teste.

\section{Discussão}

A adequação cultural de um instrumento visa produzir um texto que possibilite a utilização na cultura alvo, de modo a se manter a interação entre pesquisador e usuário, de forma tão espontânea e natural como o instrumento original em sua respectiva cultura (CECILIO, 2016).

Tem-se, como meta, a validação das traduções de instrumentos desenvolvidos para populações norte-americanas ou europeias, de forma a obter-se a melhor adaptação para a cultura brasileira (PICON et al., 2005).

Uma avaliação meticulosa da equivalência entre o instrumento original e sua versão adaptada é necessária antes de aplicar um instrumento desenvolvido para um contexto cultural com suas diferenças de definições, crenças e comportamentos. Essa adaptação transcultural enfatiza a equivalência semântica em vez de equivalência literal dos termos, com o propósito de expressar conceitos usuais na população-alvo (SARDINHA et al., 2010).

Não há um consenso sobre como adaptar um instrumento para uso em outro contexto cultural. Tal procedimento vai depender das características do instrumento, dos contextos de sua aplicação tanto na versão original como na adaptação. No entanto, é consenso que o processo de adaptação vai além da mera tradução, a qual não garante a validade de constructo, tampouco a confiabilidade da medida (BORSA; DAMÁSIO; BANDEIRA, 2012).

O alto índice de concordância nas diferentes etapas do estudo pode estar relacionado ao longo tempo de experiência teórica e prática na área de atividade física adaptada dos 
http://dx.doi.org/10.5902/1984686X41214

participantes da primeira e terceira etapa do estudo. Para Braccialli, a experiência na área de conhecimento e o domínio na língua original do instrumento contribuem para uma avaliação mais precisa na tradução e dentro do contexto do instrumento para a nova cultura na qual será utilizado (BRACCIALLI, 2017).

Os resultados do estudo indicaram que o instrumento PASIPD BR tem validade de conteúdo (CUNHA et al., 2016; MEDEIROS et al., 2015), validade transcultural (SOUZA, ALEXANDRE, GUIRARDELLO, 2017) e validade semântica (PASQUALI, 2010).

A validade de conteúdo é realizada por meio de uma avaliação qualitativa durante o processo de adaptação cultural de um instrumento já existente em outra língua. A validação é obtida por intermédio do julgamento realizado por um comitê de especialistas, que avaliam o grau de relevância, aceitabilidade, compreensão e clareza dos itens e identificam discrepâncias e omissões no questionário. Nessa etapa, é importante que o comitê de juízes tenha qualificações para realizar essas atividades, experiência na área prática e em pesquisa e conheça a metodologia utilizada. Um índice de concordância entre juízes igual ou superior a $80 \%$ indica que o instrumento tem validade de conteúdo (CUNHA et al, 2016). Os resultados dessa etapa indicaram que o instrumento tem boa validade de conteúdo, pois, o índice de concordância entre os juízes, na maior parte dos itens foi igual ou superior a 80\%, e o comitê de juízes tinha as qualificações necessárias.

$\mathrm{Na}$ validação transcultural, busca-se evidências que indicam que o instrumento traduzido e adaptado culturalmente é equivalente ao original. Para que haja validação transcultural é necessária a realização das seguintes etapas: os itens devem ser traduzidos e retrotraduzidos por tradutores independentes, a tradução deve ser revisada por um comitê de especialistas e deve ser realizado um pré-teste (SOUZA, ALEXANDRE, GUIRARDELLO, 2017). Todas as etapas foram realizadas no processo de tradução e adaptação cultural do instrumento PASIPD para a língua portuguesa, portanto, o mesmo apresenta validade transcultural.

A validação semântica envolve a compreensão, tanto a inteligibilidade, quanto a relevância dos itens do instrumento pela população-alvo. Em relação à inteligibilidade, os itens devem ser compreendidos pela população com o menor nível de habilidade (PASQUALI, 2010). Nessa etapa é necessária a realização de um pré-teste com uma amostra representativa do público-alvo. O pré-teste foi realizado e o índice de concordância foi superior a $80 \%$, exceto em uma questão, que foi adequada posteriormente, o que indica que a validade semântica do instrumento. 
http://dx.doi.org/10.5902/1984686X41214

\section{Conclusões}

O instrumento foi adequadamente traduzido e adaptado para a cultura brasileira e tem uma viabilidade para aplicação em adultos com deficiência física de forma rápida e fácil. No entanto, considera-se importante verificar a confiabilidade e validade de constructo do instrumento com a população brasileira adulta com deficiência física.

\section{Referências}

BEATON, Dorcas. et al. Guidelines for the process of cross-cultural adaptation of selfreport measures. Spine (Phila Pa 1976). n.24, v.25, p.3186-91, dez.2000.

BORSA, Juliane Callegaro; DAMÁSIO, Bruno Figueiredo; BANDEIRA, Denise Ruschel. Cross-Cultural Adaptation and Validation of Psychological Instruments. Paidéia. n.53, v.22, p.423-32, set./dez.2012.

BRACCIALLI, Ana Carla. Tradução e adaptação transcultural do instrumento educational technology predisposition assessment - Et PA. Pós-Graduação em Educação (Mestrado), Faculdade de Filosofia e Ciências, Universidade Estadual Paulista - Marília. 2017.

CARROLL, Dianna D. et al. Vital Signs: Disability and Physical Activity -- United States, 2009-2012. Morb Mortal Wkly Rep. n.18, v.63, p. 407-13, maio 2014.

CASTRO, Oscar. et al. A scoping review on interventions to promote physical activity among adults with disabilities. Disabil Health J. n.2, v.11, p.174-183, abr. 2017. Disponível em: https://doi.org/10.1016/j.dhjo.2017.10.013. Acesso em: 3 set. 2019.

CECILIO, Sumaya Giarola. Adequação cultural: Etapa complementar à tradução e adaptação de instrumentos em saúde. Pós-Graduação em Enfermagem (Mestrado), Escola de Enfermagem, Universidade Federal de Minas Gerais - UFMG Belo Horizonte. 2016.

COSTER, Wendy Jane; MANCINI, Marisa Cotta. Recommendations for translation and cross-cultural adaptation of instruments for occupational therapy research and practice. Rev Ter Ocup da Univ São Paulo [Internet]. n.1, v.26, p.50-7 abr.2015. Disponível em: https://doi.org/10.11606/issn.2238-6149.v26i1p50-57. Acesso em: 13 set. 2019.

CUNHA, Cristiane Martins; DE ALMEIDA NETO, Omar Pereira; STACKFLETH, Renata. Principais métodos de avaliação psicométrica da validade de instrumentos de medida. Revista de Atenção à Saúde, v. 14, n. 47, p. 75-83, 2016.

DE GROOT, S. et al. Evaluation of the physical activity scale for individuals with physical disabilities in people with spinal cord injury. Spinal Cord [Internet]. n.7, v.48, p.542-7, 2010. Disponível em: http://www.nature.com/doifinder/10.1038/sc.2009.178. Acesso em: 1 ago. 2019. 
http://dx.doi.org/10.5902/1984686X41214

DRIGNY, Joffrey. Transcultural validation of the physical activity scale for individuals with physical disabilities (PASIPD) in French: The PASIPD-Fr (PASIPD-

Fr). In: ClinicalTrials.gov. 2019. Disponível em:

https://clinicaltrials.gov/ct2/show/NCT04061616. Acesso em 10 out. 2019.

EISENBERG, Yochai; VANDERBOM, Kerri A.; VASUDEVAN, Vijay. Does the built environment moderate the relationship between having a disability and lower levels of physical activity? A systematic review. Prev Med (Baltim) [Internet]. Elsevier Inc. v. 95, suplemento, p.75-84, fev. 2017. Disponível em:

http://dx.doi.org/10.1016/j.ypmed.2016.07.019. Acesso em: 3 set. 2019.

FAGUNDES, Antônio Jayro da Fonseca Motta. Descrição, definição, e registro de comportamento. São Paulo: Edicon, 12 ed., 1999.

GUILLEMIN, Francis; BOMBARDIER, Claire; BEATON, Dorcas. Cross-cultural adaptation of health-related quality of life measures: Literature review and proposed guidelines. $\mathbf{J}$ Clin Epidemiol [Internet]. n.12, v.46, p.1417-32, dez.1993. Disponível em:

http://linkinghub.elsevier.com/retrieve/pii/089543569390142N. Acesso em: 1 ago. 2019.

JIMENEZ-MORENO, Aura Cecilia. et al. Measuring Habitual Physical Activity in Neuromuscular Disorders: A Systematic Review. J Neuromuscul Dis. n.1, v. 4, p. 25-52, fev. 2017.

MAT ROSLY, Maziah. et al. Malaysian adaptation of the physical activity scale for individuals with physical disabilities in individuals with spinal cord injury, Disability and Rehabilitation, n.27, p.1-9, jan.2019. Disponível em:

https://doi.org/10.1080/09638288.2018.1544294. Acesso em: 1 ago. 2019.

MEDEIROS, Rosana Kelly da Silva et al. Modelo de validação de conteúdo de Pasquali nas pesquisas em Enfermagem. Revista de Enfermagem Referência, n. 4, p. 127-135, 2015.

MELCHIORS, Ana Carolina; CORRER, Cassyano Januário; FERNÁNDEZ-LLIMOS, Fernando. Tradução e Validação para o Português do Medication Regimen Complexity Index. Arq Bras Cardiol. n.4, v.89, p.210-8, out.2007.

PASQUALI, L. Instrumentação psicológica: Fundamentos e práticas. Porto Alegre, Brasil: Artmed, 2010.

PICON, Patrícia. et al. Desenvolvimento da versão em português do Social Phobia and Anxiety Inventory (SPAI). Rev Psiquiatr do Rio Gd do Sul. n.1, v.27, p.40-50, jan/abr.2005

REICHENHEIM, Michael Eduardo; MORAES, Claudia Leite. Operacionalização de adaptação transcultural de instrumentos de aferição usados em epidemiologia. Rev Saúde Pública. n.4, v.41, p.665-73, 2007.

SARDINHA, Aline. et al. Tradução e adaptação transcultural do Questionário de Atividade Física Habitual. Rev Psiq Clín. n.1, v.37, p.16-22, jan. 2010. 
SHAW, Robert B. et al. The technical quality of online leisure time physical activity resources for people with physical disabilities. Disabil Health $\mathbf{J}$ [Internet]. Elsevier Inc. n.10, v.1, p.93-9, jan. 2017. Disponível em: http://dx.doi.org/10.1016/j.dhjo.2016.09.002. Acesso em: 13 set. 2019.

SHIELDS, Nora; SYNNOT, Anneliese. Perceived barriers and facilitators to participation in physical activity for children with disability: a qualitative study. BMC Pediatrics; p. 1-10, 2016.

SILVA, Fernanda Carolina Toledo da. Nível de atividade física, participação e qualidade de vida de pessoas com deficiência física em diferentes contextos. PósGraduação em Educação (Doutorado), Faculdade de Filosofia e Ciências, Universidade Estadual Paulista - Marília. 2019.

SKATTEBOE, Sigrid. et al. One-year trajectories of motivation and physical activity in persons with disabilities. J Rehabil Med, n.48, p.371-377, 2016

SOUZA, Ana Cláudia de; ALEXANDRE, Neusa Maria Costa; GUIRARDELLO, Edinêis de Brito. Propriedades psicométricas na avaliação de instrumentos: avaliação da confiabilidade e da validade. Epidemiologia e Serviços de Saúde, v. 26, p. 649-659, 2017.

ULAŞ, Kardem; TOPUZ, Semra; DINÇ HORASAN, Gönül. The validity and reliability of the Turkish version of the Physical Activity Scale for Individuals with Physical Disabilities (PASIPD). Turk J Med Sci, n.49, p.1-6, 2019

VAN DEN BERG-EMONS, Rita J. et al. Validation of the physical activity scale for individuals with physical disabilities. Arch Phys Med Rehabil [Internet]. Elsevier Inc. n.6, v.92, p.923-8, jan.2011. Disponível em: http://dx.doi.org/10.1016/j.apmr.2010.12.006. Acesso em: 13 set. 2019.

VAN DER PLOEG, Hidde P. et al. The Physical Activity Scale for Individuals with Physical Disabilities: Test-Retest Reliability and Comparison with an Accelerometer. J Phys Act Heal [Internet]. n.1, v.4, p.96-100, jan. 2007. Diponível em:

http://journals.humankinetics.com/doi/10.1123/jpah.4.1.96. Acesso em: 10 out.2019.

WASHBURN, Richard A. et al. The physical activity scale for individuals with physical disabilities: Development and evaluation. Arch Phys Med Rehabil. n.2, v.83, p.193-200, fev. 2002.

\section{Correspondência}

Douglas Felipe Veroni Rodrigues - Avenida Maria Fernandes Cavalari, 3190 - casa 142, Jardim Cavalari, Marília, São Paulo - Brasil.

CEP: $17526-680$ 